\title{
Correction of a coronary artery aneurysm with a polytetrafluoroethylene-Covered stent: Case report
}

Fazila-Tun-Nesa Malik*, Mir Nesaruddin Ahmed, Md. Kalimuddin, Nazir Ahmed, Mohammad Badiuzzaman, Ashok Dutta, Dhiman Banik, Kabiruzzaman, Habibur Rahman, Tawfiq Shahriar Huq and Md. Forhad Jamal

Department of cardiology, National Heart Foundation Hospital \& Research Institute, Dhaka- 1216, Bangladesh

\begin{abstract}
Coronary artery aneurysmis a focal dilatation of the coronary artery. Atherosclerosis is the most common cause of coronary artery aneurysm (CAA). An essential prerequisiteto aneurysm formation is the presence ofan abnormal tunica media in the vessel wall (erosion,ulceration, or hemorrhage in the tunica media). A few data are available regarding treatment option of this rare condition. PTFE-covered stents have emerged as a new tool for thetreatment of coronary artery aneurysms. We report this rare case of right CAA treated successfully with PTFE-covered stent.
\end{abstract}

\section{Learning objective}

Coronary artery aneurysm is defined as any coronary dilatation $\geq 1.5$ times the diameter of the normal adjacent segment or the diameter of the largest coronary vessel. Coronary artery aneurysms may be asymptomatic or symptomatic. To prevent its complications (thromboembolism, rupture, or vasospasm) correction of atherosclerotic coronary artery aneurysm may be an option. Polytetrafluoroethylene (PTFE)-covered stent is effective and safe option for correcting this aneurysm.

\section{Introduction}

With the advent of coronary angiography, coronary artery aneurysm has been diagnosed in increasing frequency. CAA can also be visualized on high resolution CT, IVUS and MRI. Most cases are asymptomatic. However, in the natural history of coronary aneurysms it may be complicated by thromboembolism, rupture, or vasospasm [1]. There is no consensus on its management. Some advocate conservative management while others advocate more aggressive approach [1]. Here we present a case of right coronary artery aneurysm, which we successfully treated with a polytetrafluoroethylene (PTFE)-covered stent (Jostent; Graft Master, Germany).

\section{Case report}

A 50 years old man with a history of chest pain for three months underwent elective coronary angiography. Risk factors included smoking, hypertension, type II diabetes and dyslipidaemia. Angiography showed (Figure 1) triple vessel disease involving proximal right coronary artery ( $75 \%$ stenosis) with anuery smal dilatation in mid segment, proximal left anterior descending artery (75-80\% stenosis), distal left circumflex artery (90-95\% stenosis) and second obtuse marginal (100\% stenosis).

We decided to treat CAA with a $3.5 \times 19 \mathrm{~mm}$ polytetrafluoroethylene (PTFE)-covered stent (Jostent; Graft Master) graft. Stent was deployed at $16 \mathrm{ATM}$ with post dilatation (Figure 2). Immediately after stent deployment aneurysm was corrected. Subsequently angioplasty of all three vessels was done. Patient was discharged after two days without complication.

\section{Discussion}

Coronary artery aneurysm (CAA) is defined as any coronary dilatation $\geq 1.5$ times the diameter of the normal adjacent segment or the diameter of the largest coronary vessel. The incidence varies from $1.5 \%$ to $5 \%$ with male dominance [2]. The most frequent coronary artery involved is the RCA in $40.4 \%$ of the cases, followed by the left anterior descendingartery in $32.3 \%$ of the cases, the circumflex in $23.4 \%$, and the left main coronary artery in $3.5 \%$ of the cases [2].

Coronary aneurysms are frequently seen in association with atherosclerosis, suggesting an overlap in risk factors and pathogenesis [3]. It has been estimated that $50 \%$ of coronary aneurysms are due to atherosclerosis. The next most common cause is congenital, accounting for $20-30 \%$ of coronary aneurysms. A host of inflammatory and
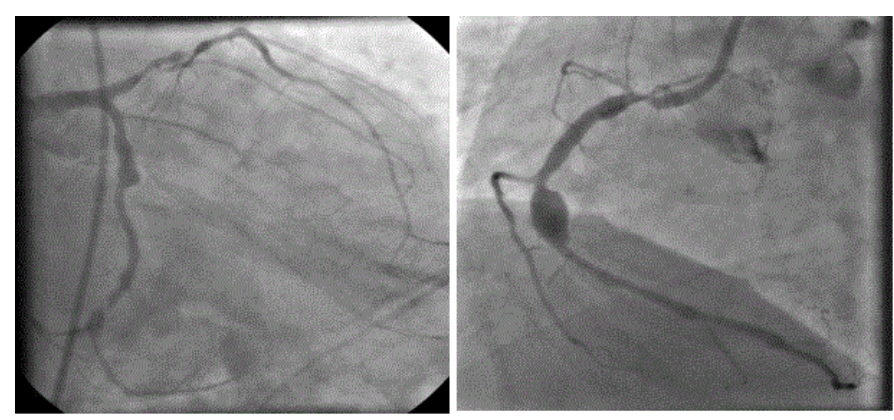

Figure 1. Triple vessel disease with aneurismal dilatation of mid segment of RCA.

Correspondence to: Fazila-Tun-Nesa Malik, Chief Consultant Cardiologist, National Heart Foundation Hospital \& Research Institute, Plot. 7/2, Section-2, Mirpur, Dhaka, Bangladesh, Tel: 880-2-9033442-6, Ext-255; Fax: 9029694; E-mail: fazilamalik@yahoo.com

Received: December 19, 2016; Accepted: January 05, 2017; Published: January 10,2017 


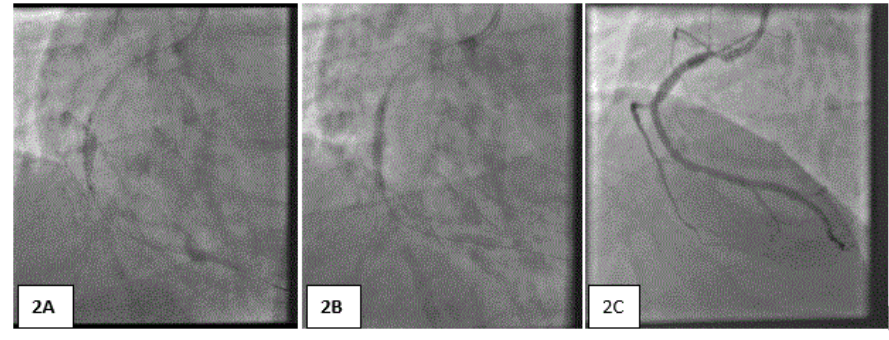

Figure 2. A: Stent placement; B: Stent deployment; C: Obliteration of aneurysm.

connective tissue disorders have also been associated with coronary aneurysms. Most well-known is the association with Kawasaki disease. Coronary aneurysms have also been reported in patients with Takayasu's arteritis, lupus, rheumatoid arthritis, Marfan syndrome, and Ehlers-Danlos syndrome. Coronary aneurysms have also been noted in conjunction with infection, drug use, trauma, and following percutaneous coronary intervention [3].

The pathogenesis for coronary artery aneurysms is usually related to the underlying cause, but an essential pre requisiteto aneurysm formation is the presence of an abnormal tunica media in the vessel wall (erosion, ulceration, or hemorrhage in the tunica media), with resultant enlargement and remodeling of an arterial segment, as clearly demonstrated in the atherosclerotic model [4].

The clinical presentation maybe varied. Most cases are asymptomatic and diagnosis is incidental during investigation for some related problem. When patients are symptomatic, the clinical manifestations depend on the underlying cause; in those cases, associated with atherosclerosis, the clinical manifestations are similar to those seen in coronary artery disease [5]. Indeed, the natural history of coronary aneurysms may be complicated by thromboembolism, rupture, or vasospasm [1]. As the incidence of each of these complications is unknown [2], the therapy for this condition is controversial. Two options are currently available for correcting coronary aneurysm [1].

First, surgery may be considered as a means to avoid complications [6], and surgery is indicated in patients with obstructive coronary artery disease or evidence of embolization leading to myocardial ischemia and in those patients with evidence of enlargement of saccular coronary artery aneurysms with increased risk of rupture [7]. Surgical management of CAA usually involves 4 types of interventions: aneurysm ligation and distal bypass; isolated coronary artery bypass grafting; resection, or marsupialization with interposition graft, and the ideal approach has not yet been formally studied [6].

Second, percutaneous treatment is a newer option that involves the placement of a covered stent to obstruct blood flow into the aneurysmal sac [8]. The synthetic membrane of the stent-graft effectively prevents plaque protrusion, successfully sealing the aneurysm-a safer and less invasive alternative in the treatment of coronary aneurysms [8]. These PTFE-covered stents, easy and rapid to deploy, have emerged as a new tool for thetreatment of coronary artery aneurysms [1]. Polytetrafluoroethylene has ideal characteristics as a single layer, and it can be rolled to form a thin multilayer covering that can be expanded 4 to 5 times its original diameter (when the stent expands) without laceration or shrinkage. Furthermore, the negative charge of the polymer prevents blood-protein coagulation on the tissue surface and limits platelet activation and thrombus formation [9].

There are two possible mechanisms by which coronary stenting might cause reduction in the size of aneurysms. One is that it decreases the velocity of coronary flow through the stenosis and attenuates the hydrodynamic wall stress on the aneurysm. The resulting decrease in hemodynamic forces could reduce the size of aneurysm [10]. A second mechanism is that stenting improves the degradation of the extracellular matrix structure through the regulation of matrix metalloproteinases(MMPs) [10].

The indication for correcting coronary artery aneurysms has been sparsely investigated and thus has a low level of evidence. It appears that the use of PTFE-covered stents is effective and safe for atherosclerotic coronary artery aneurysms.

\section{Acknowledgments}

We thank Mohammad Murshed Uddin, Chief cathlab technician and Mohammad Rasel Hossain for help with the preparation of the manuscript.

\section{Conflict of interest}

'The Authors declare that there is no conflict of interest'.

\section{References}

1. Szalat A, Durst R, Cohen A, Lotan C (2005) Use of polytetrafluoroethylene covered stent for treatmentof coronary artery aneurysm. Catheter cardiovascInterv 66: 203208 .

2. Syed M, Lesch M (1997) Coronary Artery Aneurysm: A Review. Prog Cardiovasc Dis 40:77-84.

3. Cohen P, O'Gara PT (2008) Coronary artery aneurysms: a review of the natural history, pathophysiology, and management. Cardiol Rev 16: 301-304.[Crossref]

4. Díaz-Zamudio M, Bacilio-Pérez U, Herrera-Zarza MC, Meave-González A Alexanderson-Rosas E, et al. (2009) Coronary artery aneurysms and ectasia: role of coronary CT angiography. Radiographics 29: 1939-1954.[Crossref]

5. Tunick PA, Slater J, Kronzon I, Glassman E (1990) Discrete atherosclerotic coronary artery aneurysms: a study of 20 patients. J Am Coll Cardiol 15: 279-282.[Crossref]

6. Harandi S, Johnston SB, Wood RE, Roberts WC (1999) Operative therapy of coronary arterial aneurysm. Am J Cardiol 83: 1290-1293.[Crossref]

7. LaMotte LC, Mathur VS (2000) Atherosclerotic coronary artery aneurysms: eight-year angiographic follow-up. Tex Heart Inst J 27: 72-73.[Crossref]

8. Bajaj S, Parikh R, Hamdan A, Bikkina M (2010) Covered-stent treatment of coronary aneurysm after drug-eluting stent placement: case report and literature review. Tex Heart Inst J 37: 449-454.[Crossref]

9. Di Mario C, Inglese L, Colombo A (1999) Treatment of a coronary aneurysm with a new polytetrafluoethylene-coated stent: a case report. Catheter Cardiovasc Interv 46: 463-465.[Crossref]

10. Ohtsuka M, Uchida E, Yamaguchi H, Nakajima T, Akazawa H, et al. (2007) Coronary aneurysm reduced after coronary stenting. Int J Cardiol 121: 76-77.[Crossref]

Copyright: (C2017 Malik FTN. This is an open-access article distributed under the terms of the Creative Commons Attribution License, which permits unrestricted use, distribution, and reproduction in any medium, provided the original author and source are credited. 